\title{
Completely Intrathyroidal Parathyroid Adenoma in a Patient with a Previously Failed Cervical Exploration
}

\author{
Michael Scheidt ${ }^{1}$, Daniel Hubbs ${ }^{2}$, Adam Kabaker $^{3}$, Steven De Jong ${ }^{4}$
}

\begin{abstract}
Aim: This case report highlights the importance of preoperative imaging and the clinical considerations a surgeon must have intraoperatively while searching for an ectopic parathyroid adenoma.

Background: Primary hyperparathyroidism is a disorder of autonomous function of either one or multiple parathyroid glands. Definitive treatment requires resection of the autonomous gland. Resection at the initial operation is important as reoperative patients are at a higher risk of having complications. Ectopic locations of parathyroid glands are common with a reported incidence of $6-22 \%$. Due to the greater embryonic migration distance of the inferior parathyroid glands, they have greater anatomic variation compared to the superior glands and are often more difficult to identify. True intrathyroidal parathyroid adenomas are a rare subset of ectopic parathyroids that must be considered when a parathyroid adenoma cannot be identified.

Case: A rare case of a completely intrathyroidal parathyroid adenoma in a patient with a previously failed cervical neck exploration.

Conclusion: This case demonstrates that in the setting of inconclusive imaging, one's clinical acumen, and anatomical and embryologic knowledge must be utilized in a systematic approach to identifying parathyroid adenomas. After other ectopic locations are excluded, a thyroid lobectomy should be considered to treat a completely intrathyroidal parathyroid adenoma.

Clinical significance: Anatomical variation should always be accounted for when faced with a missing parathyroid gland.

Keywords: Ectopic parathyroid, Hyperparathyroidism, Intrathyroidal parathyroid, Parathyroidectomy.

World Journal of Endocrine Surgery (2020): 10.5005/jp-journals-10002-1307
\end{abstract}

\section{INTRODUCTION}

Primary hyperparathyroidism ( $\left.1^{\circ} \mathrm{HPTH}\right)$, a disorder of autonomous function of either one or multiple glands, is classically associated with inappropriately elevated calcium and parathyroid hormone (PTH) levels. Definitive treatment of $1^{\circ} \mathrm{HPTH}$ requires surgical intervention, with resection of the autonomous gland(s), or resection of 3.5 glands in cases of uniform parathyroid enlargement. Diagnostic imaging is useful before cervical exploration with parathyroidectomy to attempt to localize the abnormal glands, including those found in ectopic locations. Some commonly utilized modalities include ultrasound (US), technetium 99m (Tc $99 \mathrm{~m}$ ) sestamibi scintigraphy (MIBI) with single-photon emission computed tomography (SPECT), SPECT/CT (MIBI SPECT fused with $\mathrm{CT}$ imaging), or parathyroid four-dimensional (4D) CT. When there is a concern for an ectopic parathyroid adenoma, surgeons need to utilize imaging and their knowledge of parathyroid embryology to systematically assess each potential ectopic location to identify the abnormal parathyroid gland and thus avoid future reoperative surgery.

During embryogenesis, the inferior and superior parathyroid glands derive from the third and fourth pharyngeal pouches, respectively. The inferior parathyroid gland, originating from the dorsal tissue of the third pharyngeal pouch, travels inferiorly with the thymus gland. While the thymus gland travels further toward the anterior mediastinum, the inferior parathyroid glands normally rest dorsally to the inferior thyroid poles. Conversely, the superior parathyroid glands migrate from the fourth pouch with the ultimobranchial bodies, descending caudally, though to a much shorter distance compared to the inferior gland counterpart. This shorter distance contributes to a more consistent location of the
'Loyola University Chicago, Stritch School of Medicine, Maywood, Illinois, USA

${ }^{2-4}$ Department of Surgery, Loyola University Medical Center, Maywood, Illinois, USA

Corresponding Author: Michael Scheidt, Loyola University Chicago, Stritch School of Medicine, Maywood, Illinois, USA, Phone: +1 414-6309640, e-mail: mscheidt@luc.edu

How to cite this article: Scheidt M, Hubbs D, Kabaker A, etal. Completely Intrathyroidal Parathyroid Adenoma in a Patient with a Previously Failed Cervical Exploration. World J Endoc Surg 2020;12(3):136-139.

Source of support: Nil

Conflict of interest: None

superior parathyroid glands, naturally posterior to the middle third of either thyroid lobe near the cricothyroid junction. ${ }^{1-4}$

Anatomic variations of parathyroid location occur when there is aberrant migration of the glands away from their normal anatomic locations. The prevalence and anatomical locations of ectopic glands have been well documented in the literature, with studies demonstrating an incidence of $6-22 \%,{ }^{1,3-7}$ Ectopic superior glands are commonly found retroesophageal or within the tracheoesophageal groove, with rare occurrences in the posterior mediastinum and intrathyroidal parenchyma. ${ }^{1-4,6}$ Due to the greater embryonic migration distance and their common migration with the thymic gland, inferior parathyroid glands have greater anatomic variation. They can commonly be found either intrathymic, within the anterior mediastinum or within the thyrothymic ligament. ${ }^{1,3,4,6}$ Submandibular (due to lack of descension) and intrathyroidal inferior parathyroids are also 

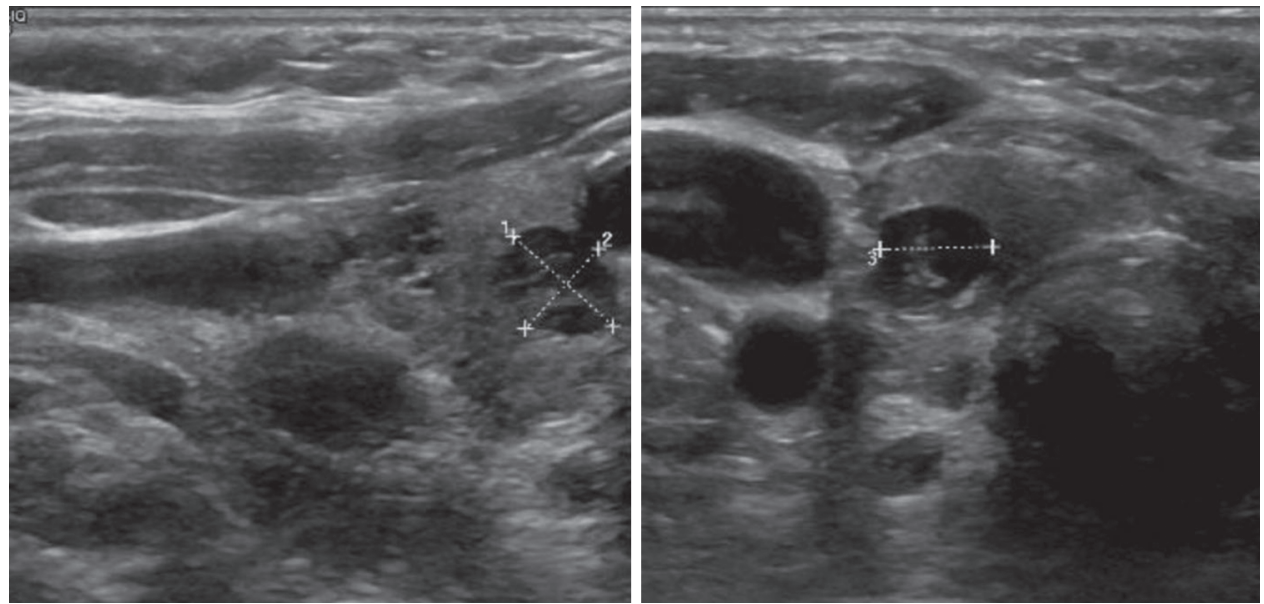

Figs $1 \mathrm{~A}$ and B: Longitudinal and transverse ultrasound images of the right lower pole of the thyroid. Imaging demonstrated a hypoechoic nodule with internal echogenic reflectors measuring approximately $1.0 \times 0.8 \times 0.8 \mathrm{~cm}$ within the right lower pole of the thyroid. Complete ultrasound exam of the thyroid demonstrated no visualization of parathyroid anatomy per the radiologic report

known variations, though to a less extent. ${ }^{1-4}$ The following case illustrates the treatment of $1^{\circ} \mathrm{HPTH}$ in a patient with a previously failed parathyroidectomy due to a missed intrathyroidal parathyroid adenoma.

\section{Case Description}

A 66-year-old woman with a past medical history of $1^{\circ} \mathrm{HPTH}$ presented to the clinic with persistent symptomatic hypercalcemia. The patient's labs had noted hypercalcemia for the last 5-6 years, and she endorsed symptoms of urinary frequency, constipation, dyspepsia, and right knee and ankle discomfort. The patient denied any history of nephrolithiasis or bone fractures, however, a previous DEXA bone density study revealed osteopenia. Initial imaging at an outside hospital (OSH) with the US demonstrated a subcentimeter right thyroid lobe nodule and no extrathyroidal nodules suspicious for parathyroid glands. Subsequent MIBISPECT/CT imaging demonstrated delayed washout within the right thyroid lobe, however, the radiologic report read negative for parathyroid disease. The patient underwent right neck exploration for $1^{\circ} \mathrm{HPTH}$ at an OSH and, despite extensive right cervical dissection, no enlarged parathyroid tissue was identified. The left neck was not explored during the procedure due to concerns of right recurrent laryngeal nerve injury. The patient subsequently underwent a direct laryngoscopy that confirmed the right vocal cord paresis.

Labs obtained before the clinic visit were significant for elevated calcium of 10.6 and a PTH level of 106, confirming persistent primary hyperparathyroidism. Repeat imaging was performed, including US (Fig. 1) and MIBI-SPECT/CT (Fig. 2). The US re-demonstrated a subcentimeter right lower pole hypoechoic thyroid nodule and the MIBI-SPECT/CT noted a delayed washout of contrast within the right lower thyroid in the vicinity of the nodule. Once again, imaging was read as negative for parathyroid disease on the radiologic report. However, the delayed wash-out on MIBI imaging was concerning to the surgery team for intrathyroidal parathyroid disease. Treatment options were discussed, and the patient elected for cervical re-exploration with subtotal parathyroidectomy and possible subtotal thyroidectomy. The patient underwent a repeat direct laryngoscopy before the surgery and the right vocal cord paresis had completely resolved.
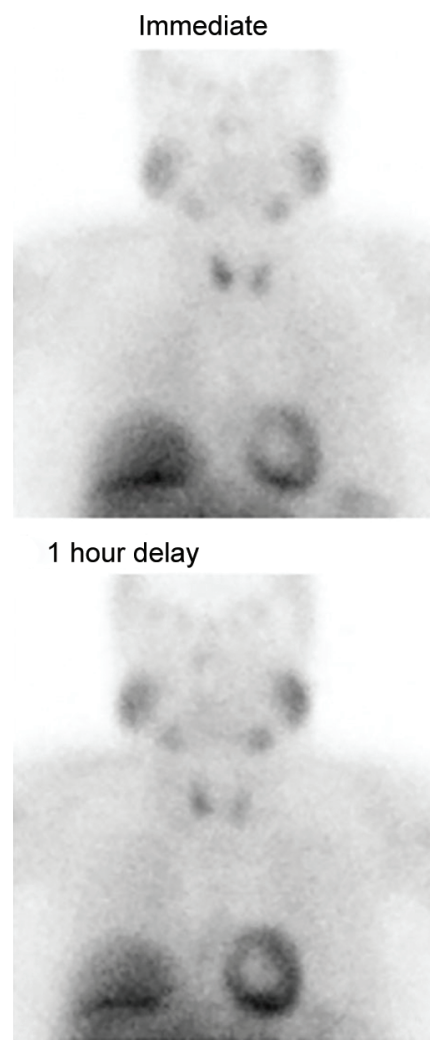

Fig. 2: Preoperative MIBI-SPECT/CT imaging demonstrating increased [99mTc]Tc-Sestamibi tracer uptake within the right inferior thyroid. Immediate planar images of the anterior neck demonstrated asymmetrical increased uptake in the right thyroid lobe compared to the left. Delayed images demonstrated incomplete tracer washout of tracer from the thyroid gland with diffuse asymmetric increased uptake within the right thyroid lobe compared to the left (red arrow). Initial radiologic reports suggested the asymmetry in uptake to be secondary to a right inferior thyroid nodule, which was later determined to be complete intrathyroidal parathyroid intraoperatively

The patient was placed in a modified Fowler position, and a transverse cervical curvilinear incision was made. The right neck was initially approached due to the findings noted on previous 
imaging. Operative findings were significant for extensive scarring secondary to the previous surgery. The right superior parathyroid was identified in its normal anatomic location and was noted to be of normal size. The right inferior parathyroid was not observed in its normal location or the typical ectopic locations. Therefore, a right thyroid lobectomy was performed and gross examination revealed a rare completely intrathyroidal parathyroid gland (Fig. 3). This finding was confirmed using an intraoperative frozen section. Intraoperative PTH levels demonstrated a significant drop from 241 to $16 \mathrm{pg} / \mathrm{mL}$ after the procedure. Postoperatively, the patient recovered without complication and the serum calcium returned to within the normal range.

\section{Discussion}

This case illustrates a rare completely intrathyroidal parathyroid adenoma in a patient with a previous failed cervical exploration. True intrathyroidal parathyroid adenomas represent a small subset of ectopic parathyroid disease with incidences ranging in the literature from 1.3 to $6.3 \% .{ }^{4,8-13}$ A retrospective study by Goodman

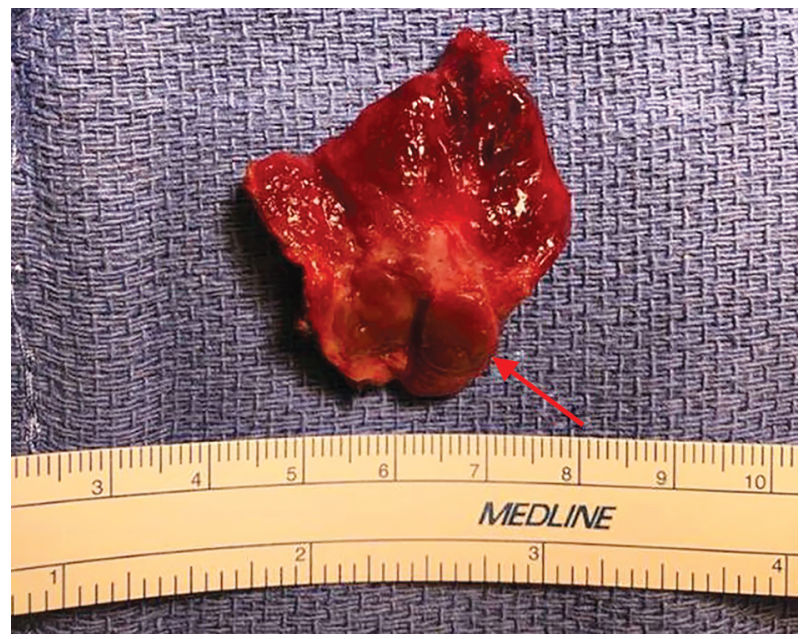

Fig. 3: Intraoperative gross anatomical image demonstrating a completely intrathyroidal parathyroid adenoma (red arrow) et al. of 11,163 patients that underwent parathyroid surgery for $1^{\circ} \mathrm{HPTH}$ categorized intrathyroidal parathyroid adenomas into three types: Type I, with the adenoma present under the thin connective tissue but not present within the thyroidal parenchyma (thus, not true intrathyroidal parathyroid); Type II, with the adenoma partially within the thyroid tissue; and Type III, with a completely intrathyroidal parathyroid adenoma (Fig. 4). ${ }^{10}$ Type I intrathyroidal parathyroid glands had the highest incidence (2.4\%), followed by Type II (1.2\%) and Type III (0.7\%). ${ }^{10}$ Ninety percent of intrathyroidal parathyroid adenomas were noted within the inferior thyroid lobe, with rare occurrences superiorly. ${ }^{10}$ This case illustrates the unusual Type III intrathyroidal adenoma requiring a partial thyroidectomy (Fig. 3). Intraprocedurally, meticulous dissection of tissues surrounding the thyroid can aid in the identification of Type I and II intrathyroidal parathyroids. However, the identification of Type III intrathyroidal adenomas is particularly reliant on accurate pre-surgical imaging or preemptive thyroidectomy when ectopic glands cannot be identified.

For our study, preoperative imaging with US and MIBI-SPECT/ $\mathrm{CT}$ was utilized. Given the previously failed cervical procedure with recurrent laryngeal nerve injury, appropriate identification of abnormal adenoma location was key to reduce the risk of complications. Ultrasound and MIBI-SPECT/CT imaging identified a $1 \mathrm{~cm}$ hypodense mass located in the right inferior thyroid lobe, with delayed washout of the tracer. Interpretation by the radiologist suggested no presence of parathyroid disease, but rather imaging consistent with a thyroid nodule. Fine needle aspiration (FNA) of the thyroidal mass was considered with washing for PTH; however, this was not performed due to its small size and risks associated with parathyroid biopsy, including lesion disruption and secondary parathyroid adenomas secondary to seeding along the needle tract. $^{14-17}$

A meta-analysis conducted by Cheung et al. assessed the accuracy of preoperative imaging on localization of parathyroid adenomas compared to intraoperative findings. ${ }^{18}$ Pooled analysis indicated that the US had a sensitivity of $76.1 \%$ (57-89\%), followed by MIBI-SPECT 79\% (49-91\%) and 4DCT (89\%). ${ }^{18}$ Data related to MIBI-SPECT/CT were not presented, though SPECT/CT is considered to have a greater localization value than SPECT alone for ectopic adenomas. ${ }^{19}$ Conversely, the positive predictive value of parathyroid
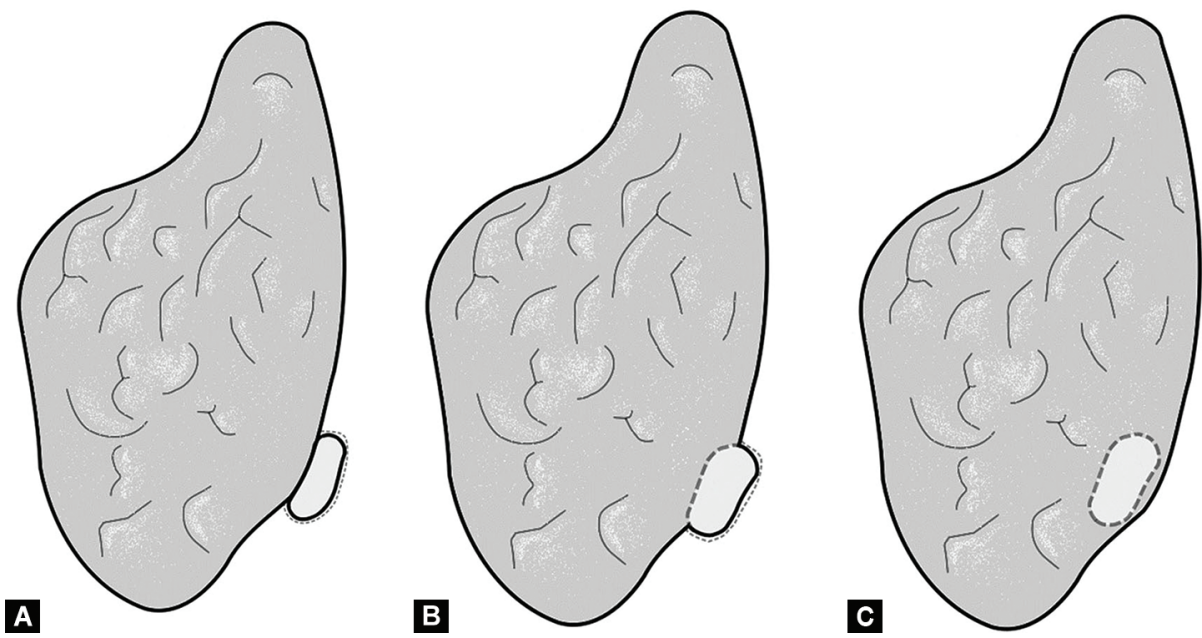

Figs 4A to C: Drawing depicting types of intrathyroidal parathyroid based upon the classification by Goodman et al. ${ }^{10}$ The classification includes Type I (A), with the adenoma present under the thin connective tissue but not present within the thyroidal parenchyma (thus, not true intrathyroidal parathyroid); Type II (B), with the adenoma partially within the thyroid tissue; and Type III (C), with a completely intrathyroidal parathyroid adenoma 
adenomas noted on imaging is similar across each test, with 4DCT having the highest PPV of $93.5 \%$, followed by US (93.2\%; 85-100\%) and $\mathrm{MIBI}$ scans $(91 \% ; 84-96 \%) .{ }^{18}$ Furthermore, a retrospective study by Roy et al. found similar results while assessing the sensitivity and PPV of MIBI scans alone and the US in the identification of ectopic parathyroid adenomas. ${ }^{7}$ Overall results determined MIBI imaging to have an overall sensitivity and PPV of 89 and $90 \%$, respectively, while the US demonstrated a sensitivity and PPV of 59 and $90 \%$, respectively. ${ }^{7}$ While assessing for intrathyroidal parathyroid adenomas only, the sensitivity of MIBI scans and the US was 78 and $83 \%$, respectively. ${ }^{7}$ The greater sensitivity of US with intrathyroidal parathyroid adenomas could be related to accessibility with the US probe, while other ectopic locations including the thymus $(48 \%$ sensitivity) or retroesophageal sites (58\% sensitivity) are much more difficult to image. ${ }^{7}$ Conversely, [99mTc]Tc-Sestamibi tracer uptake within thyroid nodules is documented within the literature, ${ }^{20}$ which can lead to misinterpretation of thyroid nodules as parathyroid disease, and vice versa. This case report demonstrated the presence of a subcentimeter thyroid nodule on the US along with delayed [99mTc] Tc-Sestamibi tracer washout, read as thyroidal nodularity on the radiologic report. In these instances, it is imperative that a surgeon creates their own interpretation of the data and considers the full clinical picture.

In conclusion, this is a case that demonstrates the treatment of $1^{\circ} \mathrm{HPTH}$ in a patient found to have an intrathyroidal parathyroid adenoma on reoperative cervical exploration. These findings demonstrate that in the setting of inconclusive imaging, one's clinical acumen and anatomical and embryologic knowledge should be utilized in a systematic approach to identifying parathyroid adenomas. Though rare, anatomical anomalies do exist, and should always be accounted for when faced with a missing parathyroid gland.

\section{References}

1. Akerstrom G, Malmaeus J, Bergstrom R. Surgical anatomy of human parathyroid glands. Surgery 1984;95(1):14-21.

2. Johnson NA, Tublin ME, Ogilvie JB. Parathyroid imaging:technique and role in the preoperative evaluation of primary hyperparathyroidism. AJR Am J Roentgenol 2007;188(6):1706-1715. DOI: 10.2214/ AJR.06.0938.

3. Solorzano CC, Carneiro-Pla D. Minimizing cost and maximizing success in the preoperative localization strategy for primary hyperparathyroidism. Surg Clin North Am 2014;94(3):587-605. DOI: 10.1016/j.suc.2014.02.006.

4. Wang C. The anatomic basis of parathyroid surgery. Ann Surg 1976;183(3):271-275. DOI: 10.1097/00000658-197603000-00010.
5. Carter WB, Carter DL, Cohn HE. Cause and current management of reoperative hyperparathyroidism. Am Surg 1993;59(2):120-124.

6. Phitayakorn R, McHenry CR. Incidence and location of ectopic abnormal parathyroid glands. Am J Surg 2006;191(3):418-423. DOI: 10.1016/j.amjsurg.2005.10.049.

7. Roy $\mathrm{M}$, Mazeh $\mathrm{H}$, Chen $\mathrm{H}$, et al. Incidence and localization of ectopic parathyroid adenomas in previously unexplored patients. World J Surg 2013;37(1):102-106. DOI: 10.1007/s00268-012-1773-z.

8. Bahar G, Feinmesser R, Joshua B, et al. Hyperfunctioning intrathyroid parathyroid gland: a potential cause of failure in parathyroidectomy. Surgery 2006;139(6):821-826. DOI: 10.1016/j.surg.2005.11.009.

9. Feliciano DV. Parathyroid pathology in an intrathyroidal position. Am J Surg 1992;164(5):496-500. DOI: 10.1016/s0002-9610(05)81188-2.

10. Goodman A, Politz D, Lopez J, et al. Intrathyroid parathyroid adenoma: incidence and location--the case against thyroid lobectomy. Otolaryngol Head Neck Surg 2011;144(6):867-871. DOI: 10.1177/0194599811400366.

11. McIntyre Jr RC, Eisenach JH, Pearlman NW, et al. Intrathyroidal parathyroid glands can be a cause of failed cervical exploration for hyperparathyroidism. Am J Surg 1997;174(6):750-754. DOI: 10.1016/ s0002-9610(97)00190-6.

12. Proye C, Bizard JP, Carnaille B, et al. Hyperparathyroidism and intrathyroid parathyroid gland. 43 cases. Ann Chir 1994;48(6):501506.

13. Wang C. Hyperfunctioning intrathyroid parathyroid gland: a potential cause of failure in parathyroid surgery. J R Soc Med 1981;74(1):49-52. DOI: 10.1177/014107688107400109.

14. Agarwal G, Prasad KK, Kar DK, et al. Indian primary hyperparathyroidism patients with parathyroid carcinoma do not differ in clinicoinvestigative characteristics from those with benign parathyroid pathology. World J Surg 2006;30(5):732-742. DOI: 10.1007/ s00268-005-0366-5.

15. Erovic BM, Harris L, Jamali $M$, et al. Biomarkers of parathyroid carcinoma. Endocr Pathol 2012;23(4):221-231. DOI: 10.1007/s12022012-9222-y.

16. Fitko R, Roth SI,Hines JR, etal. Parathyromatosis in hyperparathyroidism. Hum Pathol 1990;21(2):234-237. DOI: 10.1016/0046-8177(90)90136-s.

17. Kim J, Horowitz $G$, Hong $M$, et al. The dangers of parathyroid biopsy. J Otolaryngol Head Neck Surg 2017;46(1):4-7. DOI: 10.1186/s40463016-0178-7.

18. Cheung K, Wang TS, Farrokhyar F, et al. A meta-analysis of preoperative localization techniques for patients with primary hyperparathyroidism. Ann Surg Oncol 2012;19(2):577-583. DOI: 10.1245/s10434-011-1870-5.

19. Dasgupta DJ, Navalkissoor S, Ganatra R, et al. The role of singlephoton emission computed tomography/computed tomography in localizing parathyroid adenoma. Nucl Med Commun 2013;34(7):621626. DOI: 10.1097/MNM.0b013e3283617d5b.

20. Yordanova A, Mahjoob S, Lingohr P, et al. Diagnostic accuracy of $[(99 \mathrm{~m})$ Tc]Tc-Sestamibi in the assessment of thyroid nodules. Oncotarget 2017;8(55):94681-94691. DOI: 10.18632/oncotarget.21866. 